\title{
miR-148a and miR-375 may serve as predictive biomarkers for early diagnosis of laryngeal carcinoma
}

\author{
YING WU $^{1}$, JIA YU ${ }^{2}$, YANNI MA ${ }^{2}$, FANG WANG $^{2}$ and HONGGANG LIU ${ }^{1}$ \\ ${ }^{1}$ Department of Pathology, Beijing Key Laboratory of Head and Neck Molecular Diagnostic Pathology, \\ Beijing Tongren Hospital, Capital Medical University; ${ }^{2}$ Department of Biochemistry and Molecular Biology, \\ State Key Laboratory of Medical Molecular Biology, Institute of Basic Medical Sciences, School of Basic \\ Medicine Peking Union Medical College, Chinese Academy of Medical Sciences, Beijing 100730, P.R. China
}

Received March 7, 2015; Accepted March 18, 2016

DOI: $10.3892 / 01.2016 .4707$

\begin{abstract}
The role of microRNAs (miRs) as possible biomarkers and therapy targets has been extensively investigated in a number of types of cancer. However, the aberrant expression of miRs in laryngeal squamous cell carcinoma (LSCC), particularly during the progression of the disease, is poorly understood. In the present study, the role of miRs as possible novel early pre-diagnostic biomarkers of LSCC was investigated. TaqMan probe stem-loop quantitative polymerase chain reaction was utilized to accurately measure the amount of miR-148a and miR-375 in clinical samples of mild dysplasia, moderate dysplasia, severe dysplasia, cancer in situ, laryngeal cancer and normal epithelial controls. The application of miR-148a and miR-375 as potential predictive biomarkers for early diagnosis of LSCC was analyzed. The results of the present study suggested that miR-148a and miR-375 were significantly upregulated in LSCC tissues, and increased expression of miR-375 was associated with a more aggressive phenotype of LSCC. Additional investigation revealed that miR-148a and miR-375 increased during different dysplasia stages of LSCC carcinogenesis, and high-level expression of miR-148a or miR-375 in patients with laryngeal dysplasia may predict subsequent malignant transformation. miR-148a and miR-375 were significantly upregulated during LSCC carcinogenesis and may serve as possible predictive biomarkers for early diagnosis of LSCC.
\end{abstract}

Correspondence to: Professor Honggang Liu, Department of Pathology, Beijing Key Laboratory of Head and Neck Molecular Diagnostic Pathology, Beijing Tongren Hospital, Capital Medical University, 1 Dongjiaominxiang Street, Beijing 100730, P.R. China E-mail: liuhg1125@163.com

Abbreviations: miRs, microRNAs; LSCC, laryngeal squamous cell carcinoma; CIS, cancer in situ; ROC, receiver-operating characteristic

Key words: laryngeal squamous cell carcinoma, laryngeal precancerous lesions, tumor biomarker, microRNA-148a, microRNA-375

\section{Introduction}

Head and neck squamous cell carcinoma is the sixth most commonly observed cancer worldwide, and its mortality rate is $\sim 50 \%$ (1). Approximately 600,000 new cases are reported worldwide each year (1). Laryngeal squamous cell carcinoma (LSCC) accounts for $\sim 25 \%$ of all head and neck squamous cell carcinomas (2). Although the 5-year survival rate of LSCC is $\sim 60 \%$ (3), outcomes have not improved over the previous three decades for the majority of patients (1). One of the major reasons for the poor outcome is the low rate of diagnosis (4). The progression of LSCC may undergo several different phases: Dysplasia (including mild dysplasia, moderate dysplasia and severe dysplasia), cancer in situ (CIS) and LSCC (5). To identify novel biomarkers that indicate the early stages of LSCC, or specific biomarkers for various individuals, is urgently required for the early detection of LSCC and the development of individualized therapies. Therefore, the role of microRNAs (miRs) as possible biomarkers and targets for therapy has been extensively investigated in a number of types of cancer (6-8).

$\mathrm{miR}$ is a class of gene regulator that is able to suppress the expression of proteins via base pairing with the 3'-untranslated region of target messenger RNA (9-11). Accumulating evidence has indicated that miRs have significant roles in diverse biological processes, and the dysfunction of miRs may be implicated in a number of diseases, including cancer (12-17). Altered miR expression patterns have been reported in LSCC. For example, miR-21 and miR-106b are upregulated in LSCC cancerous tissues compared with adjacent non-tumor tissues (18). miR-34a/c, miR-370 and miR-206 have been reported to be downregulated in human LSCC tissues (19-21). miR-203 has additionally been reported to be downregulated in laryngeal squamous cell carcinoma and is able to suppress proliferation and induce apoptosis of tumors (22). However, the aberrant expression of miRs in LSCC patients and their expression during the earlier stages of the disease are poorly understood.

According to microarray data of miR expression in tumor and dysplasia tissues of 10 LSCC patients with dysplasia (23), miR-148a and miR-375 were differentially expressed in the dysplasia and tumor tissues of 1 patient. In the present study, TaqMan probe stem-loop quantitative polymerase 
chain reaction (qPCR) was utilized to accurately measure the amount of miR-148a and miR-375 in LSCC cancer tissues, CIS tissues, mild dysplasia, moderate dysplasia and severe dysplasia tissues, as well as normal controls. It was observed that miR-148a and miR-375 were significantly upregulated in LSCC tissues, and the increased level of miR-375 in LSCC was significantly associated with a more aggressive tumor phenotype. Receiver-operating characteristic (ROC) curve analysis suggested that expression levels of miR-375 may be used as markers, with high sensitivity and specificity for LSCC diagnosis. Furthermore, miR-148a and miR-375 levels increased gradually during laryngeal carcinogenesis, and an increased expression level of miR-148a or miR-375 may predict LSCC progression and could serve as an early biomarker of LSCC.

\section{Materials and methods}

Specimens. A total of 179 formalin-fixed, paraffin-embedded tissue sections were prepared from resected tissues obtained from Beijing Tongren Hospital (Beijing, China) between April 2011 and August 2014. The specimens included 29 laryngeal squamous cell carcinoma, 19 mild dysplasia, 29 moderate dysplasia, 34 severe dysplasia, 36 CIS and 32 normal controls from vocal cord polyps. The samples were obtained from 164 males and 15 females, with an average age of $57.1 \pm 0.90$ years (range, $27-86$ years). No patients had received chemoradiotherapy prior to surgery. The tumors were staged according to the revised International Union for International Cancer Control/Tumor-Node-Metastasis staging system (24), with 20 patients classified as I-II and 9 patients classified as III-IV. A total of 169 patients had no previous medical history, whilst 4 patients had a history of high blood pressure and 6 patients had a history of dyslipidemia. The present study was approved by the ethical board of Beijing Tongren Hospital.

RNA extraction. Total RNA was extracted from formalin-fixed, paraffin-embedded tissue sections with the miRNeasy FFPE kit (Qiagen China Co., Ltd., Shanghai, China) according to the manufacturer's protocol. Tissue sections were sliced (5-20 $\mu \mathrm{m}$ thick) and the first 2-3 sections were discarded. Deparaffinization solution was added to deparaffinize the paraffin-embedded tissue at $56^{\circ} \mathrm{C}$ for $3 \mathrm{~min}$, and subsequently buffer PKD was added and mixed by vortexing. Following centrifugation for $1 \mathrm{~min}$ at $11,000 \mathrm{x} \mathrm{g}, 10 \mu \mathrm{l}$ proteinase $\mathrm{K}$ was added to the lower, clear phase and incubated at $56^{\circ} \mathrm{C}$ for $15 \mathrm{~min}$, followed by incubation at $80^{\circ} \mathrm{C}$ for $15 \mathrm{~min}$ with buffer PKD. The incubation at $80^{\circ} \mathrm{C}$ in buffer PKD partially reversed formaldehyde modification of nucleic acids. The lower, clear phase was transferred into a fresh microcentrifuge tube and incubated on ice for $3 \mathrm{~min}$. The supernatant was subsequently transferred to a fresh microcentrifuge tube following centrifugation for $15 \mathrm{~min}$ at $20,000 \mathrm{x}$ g, taking care not to disturb the pellet. DNase booster buffer was added equivalent to a tenth of the total sample volume and $10 \mu \mathrm{l}$ DNase I stock solution was additionally added and incubated at room temperature for $15 \mathrm{~min}$. Buffer RBC was added to adjust the binding conditions, and ethanol (100\%) was added to the sample. Precipitates were visible following the addition of ethanol. The sample, including any precipitate, was transferred to an RNeasy MinElute spin column and centrifuged for $15 \mathrm{sec}$ at $\sim 8000 \mathrm{x}$ g, following by washing with buffer RPE. Finally, RNase-free water was directly added to the spin column membrane to elute the RNA.

Complementary DNA (cDNA) synthesis. cDNA was synthesized using Moloney Murine Leukemia Virus (M-MLV) reverse transcriptase (Invitrogen; Thermo Fisher Scientific, Inc., Waltham, MA, USA). A stem-loop reverse transcription primer was utilized for the reverse transcription of miR. Primers were designed using DNAMAN software (Lynnon Biosoft, San Ramon, CA, USA) and were synthesized by Life Technologies (Thermo Fisher Scientific, Inc.). Primer sequences are presented in Table I. Following RNase-free DNase treatment (5 U/1 $\mu \mathrm{g}$ RNA), $500 \mathrm{ng}$ total RNA was mixed with reverse transcription primer and dNTPs, incubated at $65^{\circ} \mathrm{C}$ for $5 \mathrm{~min}$ and then placed immediately on ice. Subsequently, the mixture containing reverse transcription buffer, DL-Dithiothreitol, M-MLV reverse transcriptase and RNase inhibitor was added and incubated at $37^{\circ} \mathrm{C}$ for $50 \mathrm{~min}$, followed by a final reverse transcriptase inactivation step at $75^{\circ} \mathrm{C}$ for $5 \mathrm{~min}$. cDNA samples were stored at $-80^{\circ} \mathrm{C}$ until required for PCR analysis.

qPCR assays. qPCR was performed using a CFX96 Real-Time PCR Detection System (Bio-Rad Laboratories, Inc., Hercules, CA, USA) with TaqMan probes (Applied Biosystems; Thermo Fisher Scientific, Inc.) according to the manufacturer's protocol. No cDNA template reactions were set up as negative controls and U6 snRNA was amplified as an endogenous control. All reactions were performed in triplicates. The PCR conditions were as follows: $95^{\circ} \mathrm{C}$ for $30 \mathrm{sec}$, followed by 40 cycles of $95^{\circ} \mathrm{C}$ for $5 \mathrm{sec}$ and $60^{\circ} \mathrm{C}$ for $34 \mathrm{sec}$. The experiments were repeated three times and the data were normalized using the endogenous U6 small nucleolar RNA. The $2^{-\Delta \Delta C q}$ method was utilized for the normalization of PCR data (25). Primers were designed using DNAMAN software (Lynnon Biosoft) and were synthesized by Life Technologies (Thermo Fisher Scientific, Inc.). Primer sequences are presented in Table I.

Statistical analysis. The comparison between miR-148a and miR-375 expression in laryngeal cancer, mild dysplasia, moderate dysplasia, severe dysplasia, CIS and normal epithelial tissues was evaluated using the independent samples t-test (two-tailed). Correlations of miR-148a and miR-375 expression with patient tumor stages were performed using the $t$-test followed by the Bonferroni multiple-comparison correction. $\mathrm{P} \leq 0.05$ was considered to indicate a statistically significant difference. The ROC curve analysis and all other statistical tests were performed using GraphPad Prism version 5 (GraphPad Software, Inc., La Jolla, CA, USA).

\section{Results}

miR-148a and miR-375 are significantly upregulated in $L S C C$. To accurately evaluate the expression of miR-148a and miR-375 in LSCC, qPCR using TaqMan probes was performed to measure the amount of miR-148a and miR-375. The present study initially investigated the expression of miR-148a in the laryngeal cancer tissues of 23 LSCC patients and 29 non-matched normal epithelial tissues. The expression 
Table I. Sequence of primers used in the reverse transcription-quantitative polymerase chain reaction.

\begin{tabular}{ll} 
Primer & \multicolumn{1}{c}{ Sequence $\left(5^{\prime} \rightarrow 3^{\prime}\right)$} \\
\hline miR-148a-RT & GTCGTATCCAGTGCAGGGTCCGAGGTATTCGCACTGGATACGACACAAAGT \\
miR-148a-forward & AGCTGTTCAGTGCACTACAGA \\
miR-148a-reverse & GTGCAGGGTCCGAGGT \\
miR-148a-probe & FAM-CTGGATACGACACAAAG-MGB \\
miR-375-RT & GTCGTATCCAGTGCAGGGTCCGAGGTATTCGCACTGGATACGACTCACGCG \\
miR-375-forward & CACAAAATTTGTTCGTTCGGCT \\
miR-375-reverse & GTGCAGGGTCCGAGGT \\
miR-375-probe & FAM-CTGGATACGACTCACGC-MGB \\
U6-RT & AAAATATGGAACGCTTCACGAATTTG \\
U6-forward & CTCGCTTCGGCAGCACATATACT \\
U6-reverse & ACGCTTCACGAATTTGCGTGTC \\
U6-probe & FAM-CCATGCTAATCTTCTCTGTA-MGB
\end{tabular}

miR, microRNA; RT, reverse transcription.

A

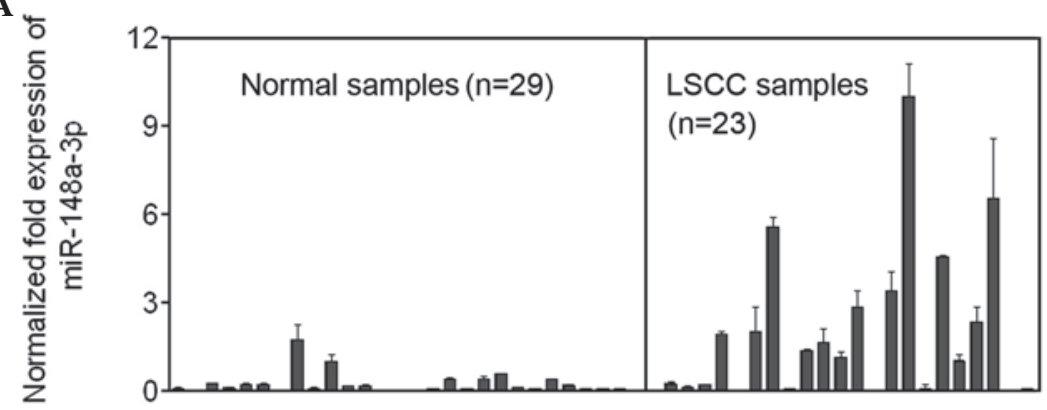

$\mathrm{C}_{4}$

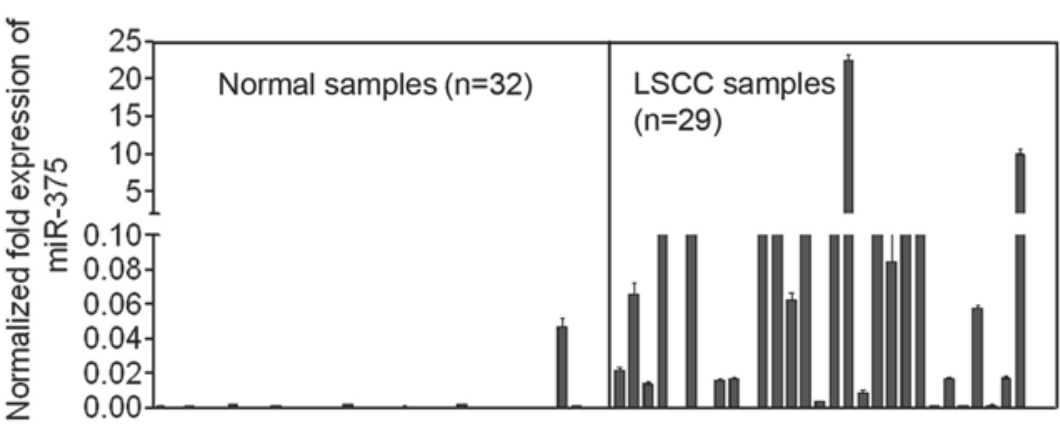

B
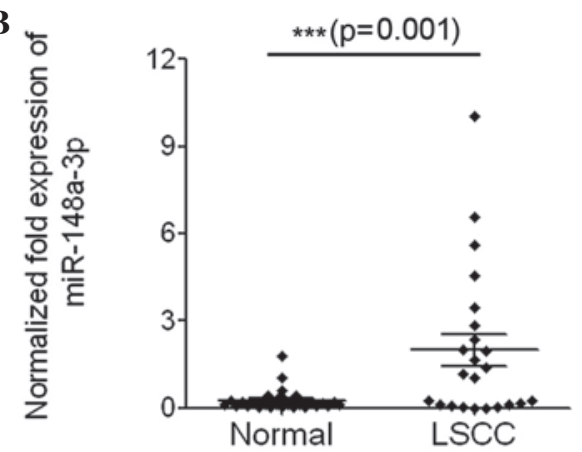

D

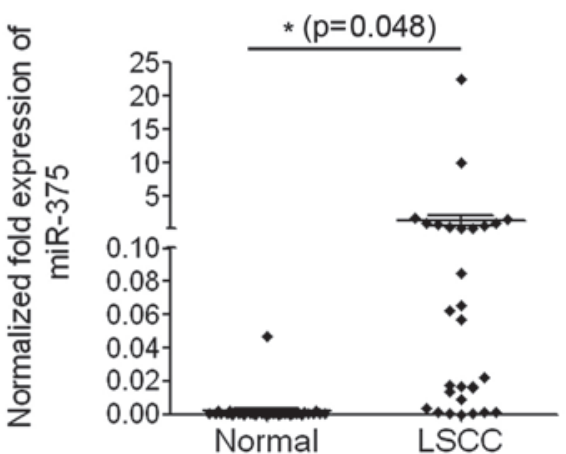

Figure 1. miR-148a and miR-375 are significantly upregulated in LSCC tissues. Normalized expression of miR-148a in laryngeal cancer tissues of 23 LSCC patients and 29 normal epithelial tissues, (A) qualified by TaqMan probe stem-loop qPCR and (B) statistically analyzed using the independent samples t-test (two-tailed). ${ }^{* * *} \mathrm{P}<0.001$. Normalized expression of miR-375 in laryngeal cancer tissues of 29 LSCC patients and 32 normal epithelial tissues, (C) qualified by TaqMan probe stem-loop qPCR and (D) statistically analyzed using the independent samples t-test (two-tailed). "P<0.05. miR, microRNA; LSCC, laryngeal squamous cell carcinoma; qPCR, quantitative polymerase chain reaction.

level of miR-148a in the majority of the laryngeal cancer tissues was increased compared with that in normal epithelial tissues, and the average expression of miR-148a in laryngeal cancer samples was significantly increased compared with that in the normal controls $(\mathrm{P}<0.001$; Fig. 1A and $\mathrm{B})$. In addition, the present study examined the expression of miR-375 in the laryngeal cancer tissues of 29 LSCC patients and 32 normal epithelial tissues. The number of clinical samples used to measure the expression levels of miR-148a and
miR-375 differed, as clinical samples with poor amplification results were not analyzed. The expression of miR-375 in normal epithelial tissues was extremely low, and its expression in the majority of laryngeal cancer tissue samples was increased compared with that in normal tissues (Fig. 1C). The average expression of miR-375 in laryngeal cancer samples was significantly increased compared with that in the normal controls ( $P=0.048$; Fig. 1D). In summary, the data indicated that miR-148a and miR-375 were significantly upregulated 
A
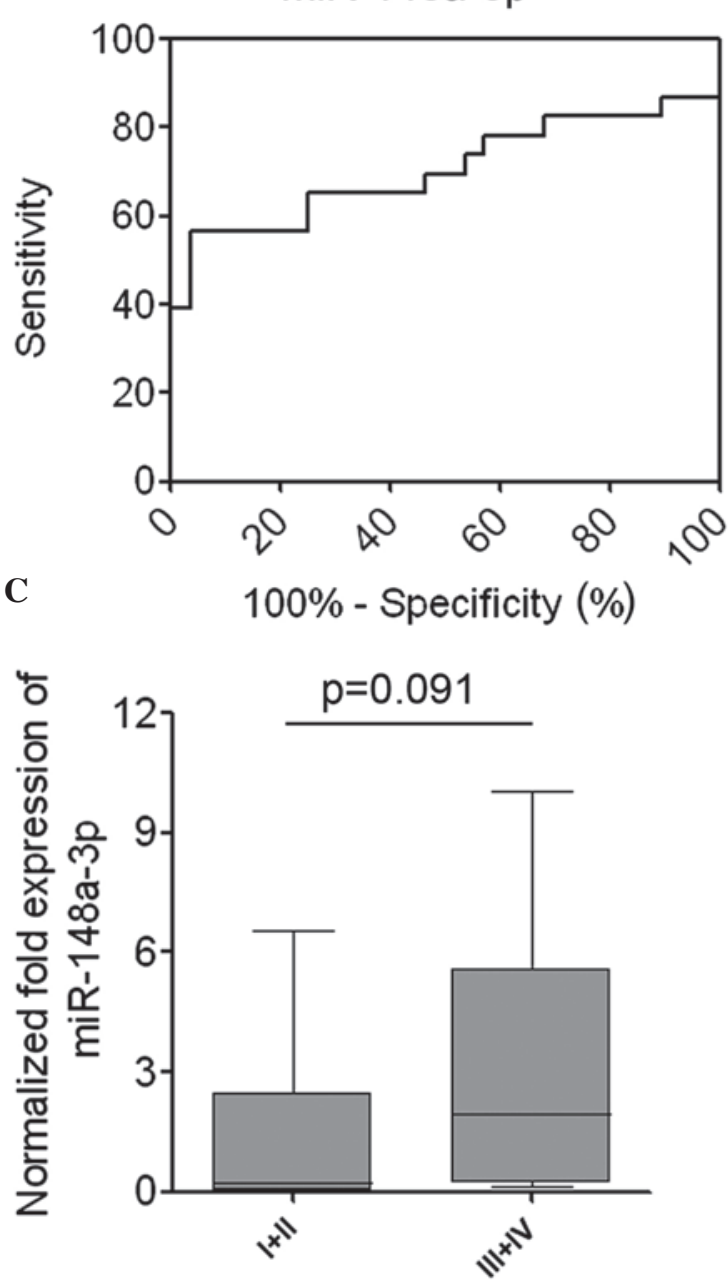
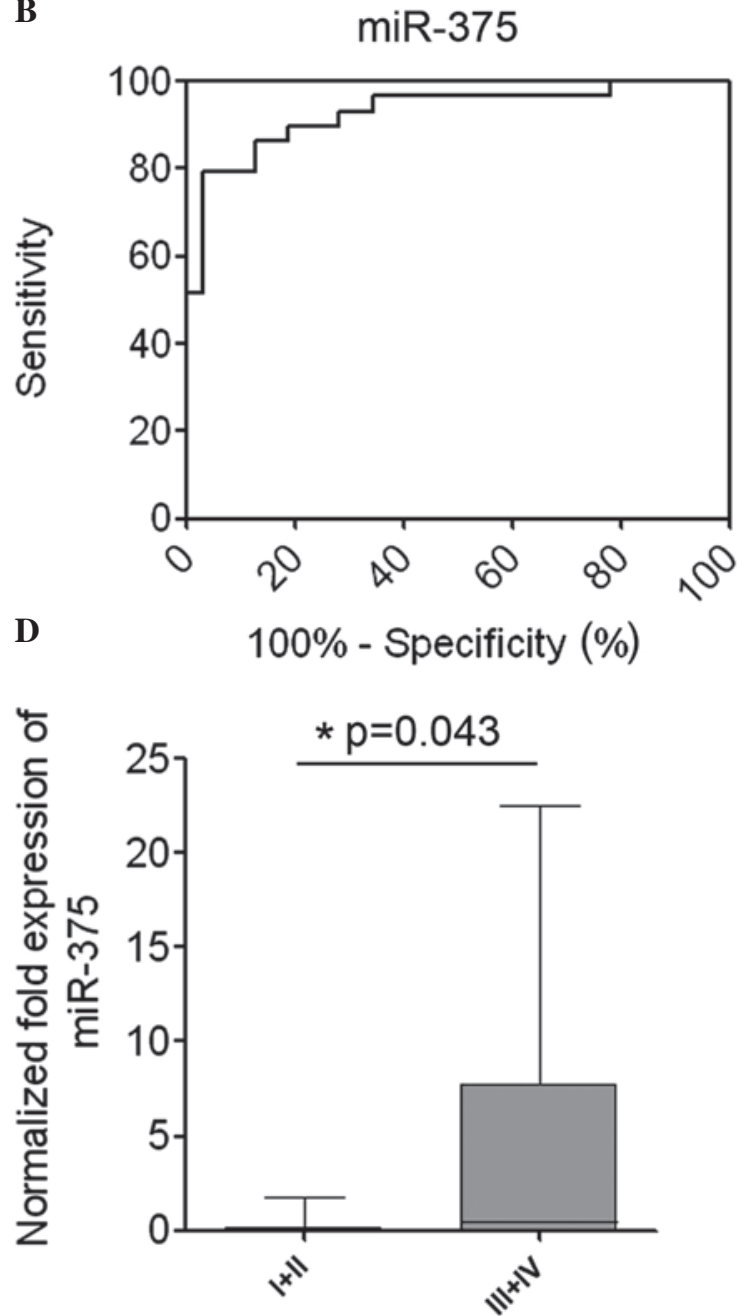

Figure 2. miR-375 may serve as a biomarker for LSCC and a high level of miR-375 is associated with aggressive phenotypes of LSCC. (A) ROC curve analysis of miR-148a as a candidate biomarker for LSCC diagnosis. AUC value, 0.7050; $\mathrm{P}=0.012$. (B) ROC curve analysis of miR-375 as a candidate biomarkers for LSCC diagnosis. AUC value, 0.927; $\mathrm{P}<0.0001$. (C) Association between the expression of miR-148a and the pTNM stage of LSCC ( $\mathrm{P}=0.091$ for stage I/II vs. III/IV). (D) Association between the expression of miR-375 and the pTNM stage of LSCC (*P<0.05 for stage I/II vs. III/IV). miR, microRNA; LSCC, laryngeal squamous cell carcinoma; ROC, receiver operating characteristic; AUC, area under the curve; pTNM, pathological Tumor-Node-Metastasis.

in laryngeal tumor tissues and may have significant roles in LSCC.

miR-375 may serve as a biomarker for LSCC diagnosis. As miR-148a and miR-375 were significantly upregulated in laryngeal cancer tissues, ROC curves were used to analyze the sensitivity and specificity of miR-148a and miR-375 as candidate biomarkers for LSCC diagnosis. The results from the test samples gave area under the curve values of 0.7050 for miR-148a $(\mathrm{P}=0.012)$ and 0.927 for miR-375 $(\mathrm{P}<0.0001)$. These results suggested that the expression levels of miR-375 may be useful as a marker, with high sensitivity and specificity for LSCC diagnosis (Fig. 2A and B).

High levels of expression of miR-148a and miR-375 are associated with aggressive phenotypes of LSCC. To additionally investigate the association between the expression of miR-148a and miR-375 and patient clinicopathological characteristics, the relative expression of miR-148a and miR-375 in LSCC tissues was statistically analyzed. Association analysis revealed that the mean expression level of miR-148a in LSCC tissues of stage III/IV was increased compared with that in tissues of stage I/II; however, this difference was not significant ( $\mathrm{P}=0.091$; Fig. 2C). High levels of miR-375 in LSCC were significantly associated with a more aggressive tumor phenotype ( $\mathrm{P}=0.043$; stage I/II vs. III/IV; Fig. 2D). The association between increased levels of miR-375 and a more aggressive phenotype of LSCC indicates that miR-375 may have a significant role in LSCC carcinogenesis.

miR-148a and miR-375 levels increase during various stages of LSCC carcinogenesis. The progression of LSCC undergoes several different phases: Dysplasia (including mild dysplasia, moderate dysplasia and severe dysplasia), CIS and LSCC (Fig. 3A). To additionally investigate the potential role of miR-148a and miR-375 in LSCC carcinogenesis, the present study measured the levels of miR-148a and miR-375 in these various phases. The present study initially examined the expression of miR-148a in 19 mild dysplasia, 26 moderate dysplasia, 32 severe dysplasia and 35 CIS tissues, and additionally compared the expression level with that in the normal controls and LSCC tissues described previously. 
A

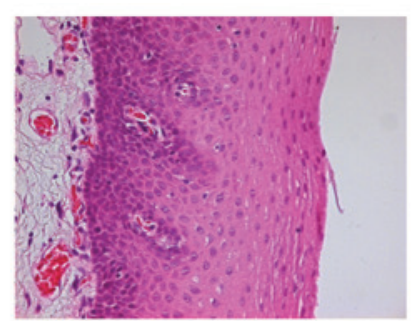

Normal

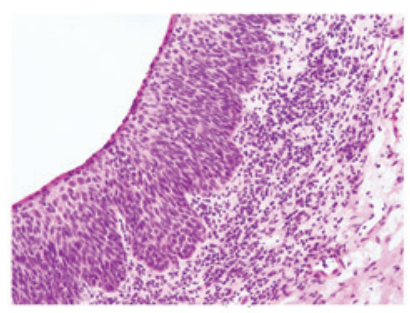

Severe dysplasia

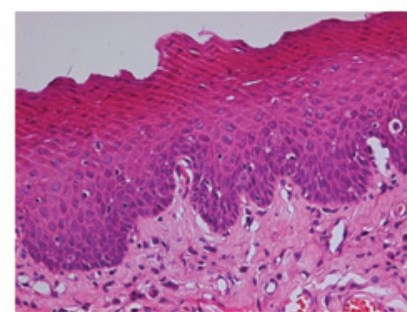

Mild dysplasia

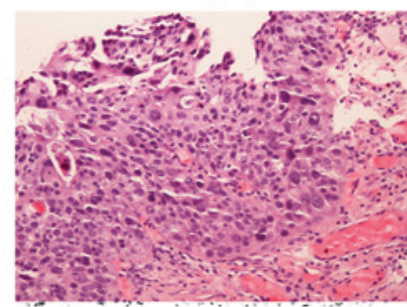

CIS

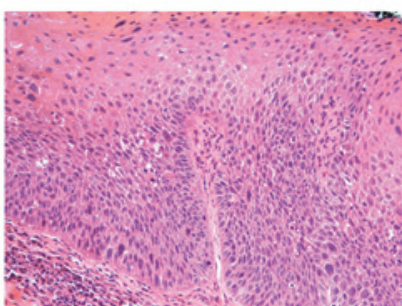

Moderate dysplasia

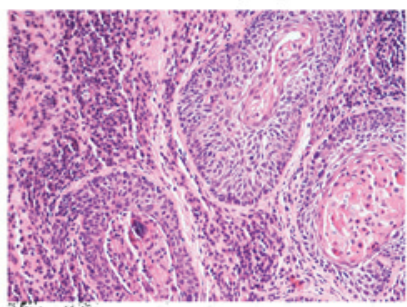

LSCC
B

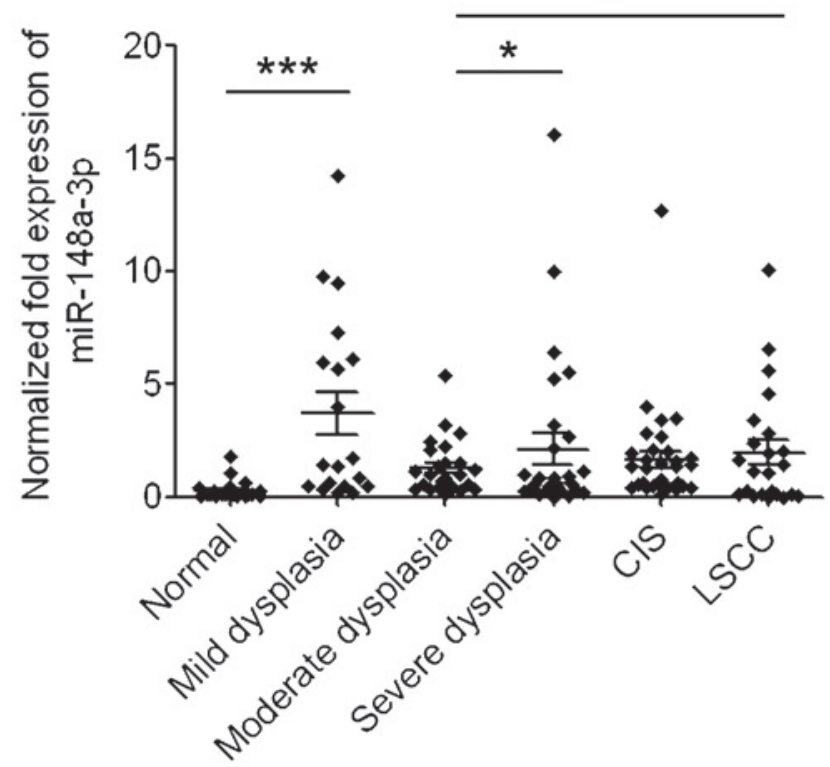

C

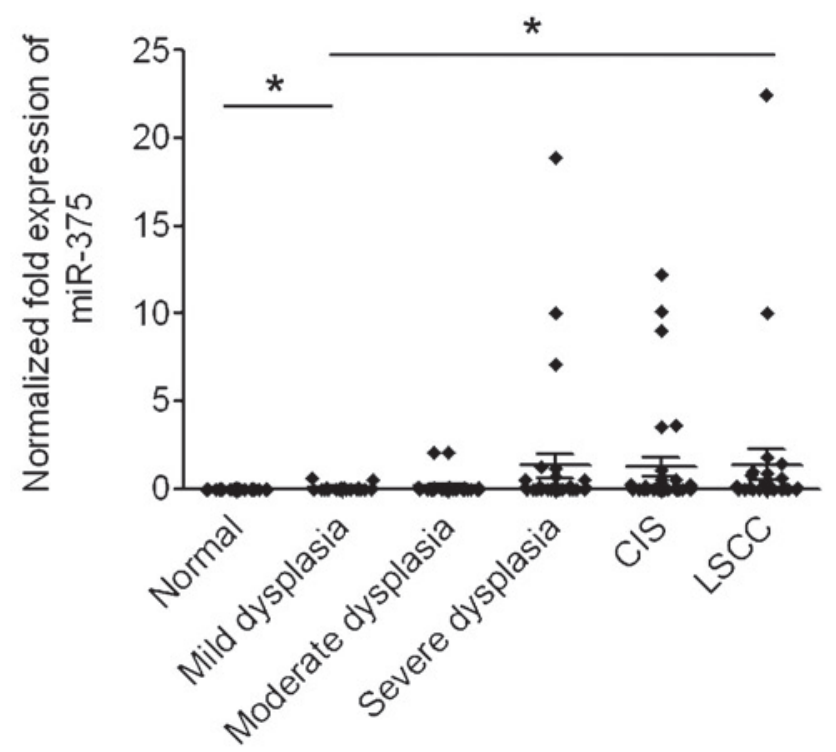

Figure 3. miR-148a and miR-375 increase during various stages of LSCC carcinogenesis. (A) Representative pathological biopsies of normal epithelial, mild dysplasia, moderate dysplasia and severe dysplasia, CIS and LSCC tissues. Hematoxylin and eosin staining; magnification, x200. (B) Normalized expression of miR-148a in normal epithelial tissues and abnormal tissues of various stages of LSCC carcinogenesis. " $\mathrm{P}<0.05$, severe dysplasia group and LSCC group vs. moderate dysplasia group; ${ }^{* * * *} \mathrm{P}<0.001$, mild dysplasia group vs. normal control group. (C) Normalized expression of miR-375 in normal epithelial tissues and abnormal tissues of various stages of LSCC carcinogenesis. "P<0.05, mild dysplasia group vs. normal control and LSCC group. miR, microRNA; LSCC, laryngeal squamous cell carcinoma; CIS, cancer in situ.

The results revealed that the expression level of miR-148a in mild dysplasia tissues was significantly increased compared with that in normal epithelial tissues $(\mathrm{P}<0.0001)$. The mean expression of miR-148a increased gradually during LSCC progression from the moderate dysplasia stage. The level of miR-148a in severe dysplasia tissues was increased compared with that in moderate dysplasia tissues $(\mathrm{P}=0.047)$; the level in LSCC was additionally increased compared with that in moderate dysplasia tissues ( $\mathrm{P}=0.049$; Fig. 3B). The expression level of miR-375 was additionally examined in 19 mild dysplasia, 29 moderate dysplasia, 34 severe dysplasia and 36 CIS tissues, and additionally compared the expression level with that in the normal controls and LSCC tissues described previously. The results revealed that the mean expression of miR-375 increased gradually during LSCC progression from dysplasia. The expression level of miR-375 in mild dysplasia tissues was significantly increased compared with that in normal epithelial tissues $(\mathrm{P}=0.027)$; the level in $\mathrm{LSCC}$ was additionally higher compared with that in mild dysplasia tissues ( $P=0.048$; Fig. $3 \mathrm{C})$. These results suggested that miR-148a and miR-375 may have significant roles in dysplasia and LSCC progression.

Positive association between the expression level of miR-148a of miR-375 and malignant transformation. To additionally investigate whether increased expression of miR-148a or miR-375 in patients with dysplasia is able to predict disease progression, the present study analyzed the expression of miR-148a and miR-375 in the dysplasia tissues of a number of malignantly transformed patients. Among the patients with 
A

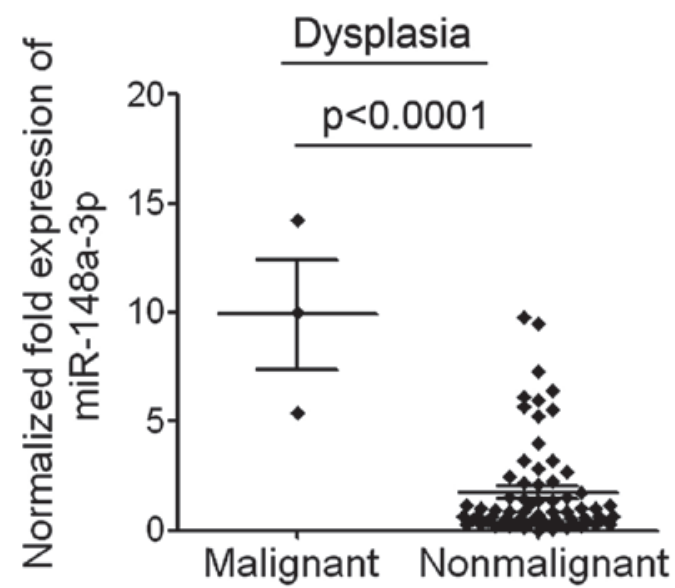

C

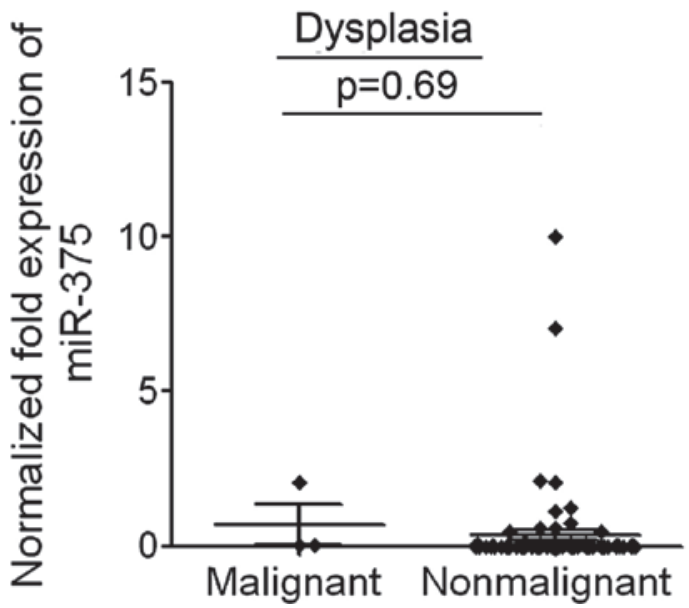

B

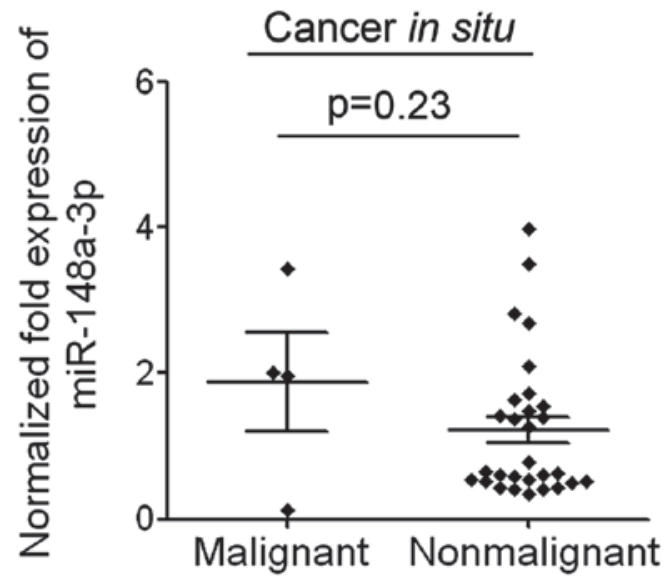

D

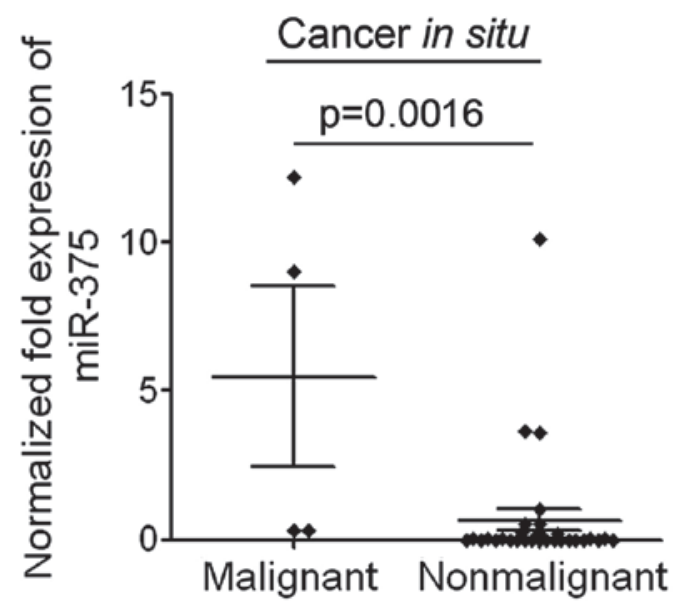

Figure 4. Expression level of miR-148a or miR-375 in dysplasia and CIS tissues with and without malignant transformation. (A) Normalized expression of miR-148a in the dysplasia tissues of 3 currently malignantly transformed patients and the remaining nonmalignantly transformed patients $(\mathrm{P}<0.0001)$. (B) Normalized expression of miR-148a in the CIS tissues of 4 currently malignantly transformed patients and the remaining nonmalignantly transformed patients $(\mathrm{P}=0.23)$. (C) Normalized expression of miR-375 in the dysplasia tissues of 3 currently malignantly transformed patients and the remaining nonmalignantly transformed patients $(\mathrm{P}=0.69)$. (D) Normalized expression of miR-375 in the CIS tissues of 4 currently malignantly transformed patients and the remaining nonmalignantly transformed patients $(\mathrm{P}=0.0016)$. miR, microRNA; CIS, cancer in situ.

dysplasia or CIS, only a small number of patients progressed to LSCC and thus, the number of malignantly transformed patients and non-malignantly transformed patients was different. The mean expression level of miR-148a in the dysplasia tissues of 3 malignantly transformed patients was significantly increased compared with that in currently nonmalignantly transformed patients $(\mathrm{P}<0.0001$; Fig. 4A). The expression level of miR-148a in CIS tissues of 4 malignantly transformed patients was also slightly increased compared with that in currently nonmalignantly transformed patients (Fig. 4B). The mean expression level of miR-375 in the dysplasia tissues of 3 malignantly transformed patients was slightly higher compared with that in currently nonmalignantly transformed patients (Fig. 4C). The expression level of miR-375 in CIS tissues of 4 malignantly transformed patients was significantly increased compared with that in currently nonmalignantly transformed patients $(\mathrm{P}=0.0016$; Fig. 4D). These results suggested that patients with increased expression of miR-148a or miR-375 may be more prone to malignant transformation. The association between the expression level of miR-148a or miR-375 and malignant transformation requires additional investigation in an increased number of clinical samples.

\section{Discussion}

In the present study, the expression of miR-148a and miR-375 was examined in a number of patients with laryngeal dysplasia or LSCC, and the potential application of the expression of these miRs in LSCC diagnosis was investigated. The expression profiles of miR-148a and miR-375 in LSCC indicated that they are significantly upregulated in LSCC, and their increased levels were additionally associated with more aggressive tumor phenotypes. Additional analysis suggested that miR-375 may serve as a potential biomarker for LSCC diagnosis. Several innate properties of miRs mean that they are attractive as potential biomarkers. miRs are small and stable against degradation and can be detected easily by specific and sensitive qPCR in small quantities of sample. miRs are additionally detectable in bodily fluids, including serum, plasma, saliva, urine and tears $(26,27)$. Furthermore, expression profiles of miRs in the plasma and/or serum of cancer patients may 
reflect the change in expression of miRs in tumor cells (28) Circulating miRs may represent a novel class of non-invasive biomarkers for cancer diagnostic and prognostic information. Therefore, the present study examined the expression levels of miR-148a and miR-375 in the serum of LSCC patients and investigated the potential application of circulating miRs in LSCC diagnosis.

The present study investigated the expression of miR-148a and miR-375 during various phases of LSCC progression and observed that miR-148a and miR-375 increased during different stages of LSCC carcinogenesis. Notably, the association analysis of $\mathrm{miR}$ expression with disease progression indicated that increased expression of miR-148a or miR-375 in patients with laryngeal dysplasia may predict subsequent malignant transformation and may therefore serve as an early biomarker of LSCC. Early diagnosis of cancer is crucial for improving cancer therapy. To intervene or administer treatment at early or precancerous stages may prevent disease progression or carcinogenesis, and is important for increasing recovery rates and improving patient quality of life $(29,30)$. Therefore, association between the expression levels of miR-148a or miR-375 and malignant transformation require additional investigation in a larger number of clinical samples and the potential application of miR-148a or miR-375 as early biomarkers of LSCC requires further investigation.

The aberrant expression of miR-148a has been reported in various forms of cancer, including hepatocellular carcinoma, bladder, breast and gastric cancer (31-34). However, no previous studies have examined the expression level of miR-148a in laryngeal carcinoma. To the best of our knowledge, the present study is the first to report the aberrant expression of miR-148a in LSCC. Recently, miR-148a was identified as a target of $\mathrm{H} 19$ and involved in regulating LSCC progression (35). The detailed function and mechanism of miR-148a in regulating LSCC progression required further investigation. The expression level of miR-375 in paired-LSCC tissues has been previously investigated (18). Yu et al (18) reported that miR-375 was downregulated in LSCC tissues, which was inconsistent with the results of the present study. This previous study compared the expression of miR-375 in LSCC tissue with adjacent normal tissues (18). Increasing evidence has suggested that normal tissues adjacent to cancer are not necessarily normal. For example, gene expression data from pancreatic cancer and adjacent normal tissue specimens demonstrated that the adjacent normal tissues had already acquired a number of transcriptional alterations and was not an appropriate baseline for comparison with cancer (36). A similar phenomenon has been observed in colorectal cancer patients (37). Therefore, the change in expression of miR-375 between LSCC tissues and adjacent normal tissues was not in accordance with that between LSCC tissues and normal laryngeal tissues. The authors of the present study propose that the expression profile of miR-375 in a number of tissues in the various phases of LSCC progression presented in the current paper may reflect the expression changes of miR-375 during LSCC progression in vivo more appropriately. The detailed function and underlying mechanism of miR-375 regulation of LSCC carcinogenesis requires additional investigation.

In conclusion, the present study determined that miR-148a and miR-375 levels are increased during LSCC progression, and high levels of expression of these two miRs was associated with aggressive phenotypes of LSCC. Notably, an increased expression level of miR-148a or miR-375 in patients with laryngeal dysplasia may predict subsequent malignant transformation, and these results suggest that miR-148a and miR-375 may serve as potential predictive biomarkers for early diagnosis of LSCC.

\section{Acknowledgements}

The present study was supported by grants from the Research Fund for the Doctoral Program of Higher Education of China, Beijing, China (grant no. 20131107110004) and the Scientific and Technological Innovation Base for Training and Development of Engineering Projects, Beijing, China (grant no. Z141107004414028).

\section{References}

1. Chen Z, Jin Y, Yu D, Wang A, Mahjabeen I, Wang C, Liu X and Zhou X: Down-regulation of the microRNA-99 family members in head and neck squamous cell carcinoma. Oral Oncol 48: 686-691, 2012.

2. Cai K, Wang Y and Bao X: MiR-106b promotes cell proliferation via targeting RB in laryngeal carcinoma. J Exp Clin Cancer Res 30: 73, 2011.

3. Ferlay J, Shin HR, Bray F, Forman D, Mathers C and Parkin DM: Estimates of worldwide burden of cancer in 2008 : GLOBOCAN 2008. Int J Cancer 127: 2893-2917, 2010.

4. Fleskens SA, Bergshoeff VE, Voogd AC, van Velthuysen ML, Bot FJ, Speel EJ, Kremer B, Takes R and Slootweg P: Interobserver variability of laryngeal mucosal premalignant lesions: A histopathological evaluation. Mod Pathol 24: 892-898, 2011.

5. Furusaka T, Susaki Y, Saito T, Katsura Y and Ikeda M: Long-term follow-up and salvage surgery in patients with T2N0M0 squamous cell carcinoma of the glottic larynx following concurrent chemoradiation therapy with cisplatin and 5-fluorouracil for laryngeal preservation. Acta Otolaryngol 133: 91-98, 2013.

6. Kimura S, Naganuma S, Susuki D, Hirono Y, Yamaguchi A, Fujieda S, Sano K and Itoh H: Expression of microRNAs in squamous cell carcinoma of human head and neck and theesophagus: miR-205 and miR-21 are specific markers for HNSCC and ESCC. Oncol Rep 23: 1625-1633, 2010.

7. Xie L, Qian X and Liu B: MicroRNAs: Novel biomarkers for gastrointestinal carcinomas. Mol Cell Biochem 341: 291-299, 2010.

8. Alencar AJ, Malumbres R, Kozloski GA, Advani R, Talreja N, Chinichian S, Briones J, Natkunam Y, Sehn LH, Gascoyne RD, et al: MicroRNAs are independent predictors of outcome in diffuse large B-cell lymphoma patients treated with R-CHOP. Clin Cancer Res 17: 4125-4135, 2011.

9. Bartel DP: MicroRNAs: Genomics, biogenesis, mechanism, and function. Cell 116: 281-297, 2004.

10. Borel C, Deutsch S, Letourneau A, Migliavacca E, Montgomery SB, Dimas AS, Vejnar CE, Attar H, Gagnebin M, Gehrig C, et al: Identification of cis- and trans-regulatory variation modulating microRNA expression levels in human fibroblasts. Genome Res 21: 68-73, 2011.

11. Macfarlane LA and Murphy PR: MicroRNA: Biogenesis, function and role in cancer. Curr Genomics 11: 537-561, 2010.

12. Yu S, Lu Z, Liu C, Meng Y, Ma Y, Zhao W, Liu J, Yu J and Chen J: miRNA-96 suppresses KRAS and functions as a tumor suppressor gene in pancreatic cancer. Cancer Res 70: 6015-6025, 2010.

13. Davidson B, Tropé CG and Reich R: The clinical and diagnostic role of microRNAs in ovarian carcinoma. Gynecol Oncol 133: 640-646, 2014.

14. Nugent M: MicroRNA function and dysregulation in bone tumors: The evidence to date. Cancer Manag Res 6: 15-25, 2014.

15. Liu C, Yu J, Yu S, Lavker RM, Cai L, Liu W, Yang K, He X and Chen S: MicroRNA-21 acts as an oncomir through multiple targets in human hepatocellular carcinoma. J Hepatol 53: 98-107, 2010.

16. Wang Y, Kim S and Kim IM: Regulation of metastasis by microRNAs in ovarian cancer. Front Oncol 4: 143, 2014. 
17. Han C, Yu Z, Duan Z and Kan Q: Role of microRNA-1 in human cancer and its therapeutic potentials. Biomed Res Int 2014: $428371,2014$.

18. Yu X, Wu Y, Liu Y, Deng H, Shen Z, Xiao B and Guo J: miR-21, miR-106b and miR-375 as novel potential biomarkers for laryngeal squamous cell carcinoma. Curr Pharm Biotechnol 15: $503-508,2014$

19. Li W, Ma H and Sun J: MicroRNA-34a/c function as tumor suppressors in Hep-2 laryngeal carcinoma cells and may reduce GALNT7 expression. Mol Med Rep 9: 1293-1298, 2014.

20. Yungang W, Xiaoyu L, Pang T, Wenming L and Pan X: miR-370 targeted FoxM1 functions as a tumor suppressor in laryngeal squamous cell carcinoma (LSCC). Biomed Pharmacother 68: 149-154, 2014.

21. Zhang T, Liu M, Wang C, Lin C, Sun Y and Jin D: Down-regulation of MiR-206 promotes proliferation and invasion of laryngeal cancer by regulating VEGF expression. Anticancer Res 31: 3859-3863, 2011.

22. Tian L, Li M, Ge J, Guo Y, Sun Y, Liu M and Xiao H: MiR-203 is downregulated in laryngeal squamous cell carcinoma and can suppress proliferation and induce apoptosis of tumours. Tumour Biol 35: 5953-5963, 2014.

23. Zhang HK and Liu HG: Is severe dysplasia the same lesion as carcinoma in situ? 10-year follow-up of laryngeal precancerous lesions. Acta Otolaryngol 2: 325-328, 2012.

24. Barnes L, Eveson JW, Reichart P and Sidransky D (eds): World Health Organization Classification of Tumours. Pathology and Genetics of Head and Neck Tumours. IARC Press, Lyon, pp10-80, 2001.

25. Livak KJ and Schmittgen TD: Analysis of relative gene expression data using real-time quantitative PCR and the 2(-Delta Delta C(T)) Method. Methods 25: 402-408, 2001.

26. Kosaka N, Iguchi $\mathrm{H}$ and Ochiya T: Circulating microRNA in body fluid: A new potential biomarker for cancer diagnosis and prognosis. Cancer Sci 101: 2087-2092, 2010.

27. Cortez MA, Bueso-Ramos C, Ferdin J, Lopez-Berestein G, Sood AK and Calin GA: MicroRNAs in body fluids - the mix of hormones and biomarkers. Nat Rev Clin Oncol 8: 467-477, 2011.
28. Taylor DD and Gercel-Taylor C: MicroRNA signatures of tumor-derived exosomes as diagnostic biomarkers of ovarian cancer. Gynecol Oncol 110: 13-21, 2008.

29. Zhu Y, Yang SR, Wang PP, Savas S, Wish T, Zhao J, Green R, Woods M, Sun Z, Roebothan B, et al: Influence of pre-diagnostic cigarette smoking on colorectal cancer survival: Overall and by tumour molecular phenotype. Br J Cancer 110: 1359-1366, 2014.

30. Nan H, Giovannucci EL, Wu K, Selhub J, Paul L, Rosner B, Fuchs CS and Cho E: Pre-diagnostic leukocyte genomic DNA methylation and the risk of colorectal cancer in women. PLoS One 8: e59455, 2013.

31. Liu L, Ye JX, Qin YZ, Chen QH and Ge LY: Evaluation of miR-29c, miR-124, miR-135a and miR-148a in predicting lymph node metastasis and tumor stage of gastric cancer. Int J Clin Exp Med 8: 22227-22236, 2015.

32. Jiang Q, He M, Ma MT, Wu HZ, Yu ZJ, Guan S, Jiang LY, Wang Y, Zheng DD, Jin F and Wei MJ: MicroRNA-148a inhibits breast cancer migration and invasion by directly targeting WNT-1. Oncol Rep 35: 1425-1432, 2016

33. Ma L, Xu Z, Xu C and Jiang X: MicroRNA-148a represents an independent prognostic marker in bladder cancer. Tumour Biol: Dec 23, 2015 [Epub ahead of print].

34. Jung KH,Zhang J,Zhou C, ShenH, GageaM,Rodriguez-Aguayo C, Lopez-Berestein G, Sood AK and Beretta L: Differentiation therapy for hepatocellular carcinoma: Multifaceted effects of miR-148a on tumor growth and phenotype and liver fibrosis. Hepatology 63: 864-879, 2016.

35. Wu T, Qu L, He G, Tian L, Li L, Zhou H, Jin Q, Ren J, Wang Y, Wang J, et al: Regulation of laryngeal squamous cell cancer progression by the lncRNA H19/miR-148a-3p/DNMT1 axis. Oncotarget 7: 11553-11566, 2016.

36. Wu Y, Wang X, Wu F, Huang R, Xue F, Liang G, Tao M, Cai P and Huang Y: Transcriptome profiling of the cancer, adjacent non-tumor and distant normal tissues from a colorectal cancer patient by deep sequencing. PLoS One 7: e41001, 2012.

37. Clare SE, Pardo I, Mathieson T, Lillemoe HA, Blosser RJ, Choi M, Sauder CAM, Doxey DK, Badve S, Storniolo AMV, et al: Abstract P1-03-02: 'Normal' tissue adjacent to breast cancer is not normal. Cancer Res 72: P1-03-02, 2012. 